\section{Zelboraf dosing rework}

\section{By Tim Fulmer, Senior Writer}

Novartis Institutes for BioMedical Research and University of California, San Francisco researchers have shown that simply altering the dosing regimen of Zelboraf is sufficient to prevent the development of drug resistance in mouse models of melanoma. ${ }^{1}$ The researchers now plan to test an intermittent dosing regimen of Zelboraf in patients with melanoma.

Zelboraf vemurafenib (PLX4032) is a small molecule BRAF inhibitor marketed by Roche and Daiichi Sankyo Co. Ltd. to treat metastatic melanoma in patients expressing the V600E BRAF mutation. That mutation occurs in about $60 \%$ of melanomas and $7 \%$ of all cancers. ${ }^{2}$ By inhibiting BRAF, Zelboraf blocks downstream activation of the MAPK pathway, which otherwise would trigger tumor cell proliferation.

About half of treated patients develop resistance to the drug in six to nine months. The mechanisms of Zelboraf resistance in tumors are still being worked out, although preclinical research has suggested multiple possible scenarios. These include the development of secondary mutations in BRAF, presence of activating mutations in downstream nodes of the BRAF pathway and activation of alternative cell survival pathways. ${ }^{3-5}$

To better understand the mechanisms of Zelboraf resistance, University of California, San Francisco (UCSF) researchers collaborated with researchers at the Novartis Institutes for BioMedical Research (NIBR) to develop an animal model of BRAF inhibitor-resistant melanoma.

The team was led by Martin McMahon, professor of cancer biology at UCSF and co-leader of the Developmental Therapeutics Program and UCSF's Hellen Diller Family Comprehensive Cancer Center, and Darrin Stuart, senior research investigator at NIBR.

The first step was generating xenograft mice with subcutaneous melanoma tumors derived from a patient expressing the V600E BRAF mutation. The animals then were treated daily with Zelboraf. After about 56 days of dosing, drug-resistant tumors developed in 2 of 10 animals.

One of those tumors was then resected, subdivided and reimplanted into a new cohort of mice, which were treated twice daily with Zelboraf to generate a model of Zelboraf-resistant melanoma.
Immunoblot analysis of the mouse tumors showed that Zelborafresistant tumors had substantially higher levels of BRAF mRNA and BRAF protein than drug-sensitive tumors, suggesting high BRAF levels contributed to Zelboraf resistance.

Curiously, when Zelboraf was discontinued in mice with drugresistant melanomas, the tumors regressed within 10 days after drug withdrawal. That result suggested Zelboraf-resistant tumors were at a growth disadvantage in the absence of drug.

Thus, although continuous Zelboraf dosing might select for drugresistant cells, intermittent dosing could create a tumor environment disadvantageous to the development of drug-resistant cells.

Indeed, mice continuously dosed developed lethal Zelboraf resistance within 100 days. In contrast, none of the mice on an intermittent dosing schedule developed drug resistance over the course of 200 days.

The authors concluded that intermittent dosing of Zelboraf "could serve to eliminate the fitness advantage of the resistant [melanoma] cells and delay the onset of drug-resistant disease."

The findings were published in Nature.

McMahon told SciBX that his lab will now collaborate with the UCSF Melanoma Center to test intermittent Zelboraf dosing in the clinic. He declined to provide a timeline for starting the study.

Zelboraf's label recommends a twice-daily $960 \mathrm{mg}$ dose until disease progression or unacceptable toxicity occurs. The label advises against reducing the twice-daily dose below $480 \mathrm{mg}$.

McMahon said that the UCSF team also will look into whether the dosing findings in melanoma can be generalized to other cancers with mutated BRAF, such as lung and thyroid cancer.

NIBR will continue to explore alternative dosing schedules as a strategy for overcoming drug resistance in patients with cancer, Stuart told SciBX. He declined to provide further details on therapeutic compounds being tested or specific cancer indications.

The mouse models developed in the Nature paper "provide a unique opportunity to study the dynamics of tumor cell populations and the evolution of resistance," added Stuart.

After Zelboraf, the most advanced BRAF inhibitor is GlaxoSmithKline plc's dabrafenib (GSK2118436). The small molecule is in registration to treat advanced or metastatic melanoma and in Phase III testing to treat solid tumors in patients with V600E BRAF mutations.

Last June, GSK presented Phase I/II data at the American Society of Clinical Oncology (ASCO) meeting showing that dabrafenib plus a MEK inhibitor prolonged progression-free survival and decreased secondary malignancies compared with Zelboraf or dabrafenib alone in patients with melanoma. MEK mediates downstream BRAF signaling.

GSK did not respond to requests for comment.

Fulmer, T. SciBX 6(5); doi:10.1038/scibx.2013.106

Published online Feb. 7, 2013 


\section{ANALYSIS}

\section{TARGETS \& MECHANISMS}

\section{REFERENCES}

1. Das Thakur, M. et al. Nature; published online Jan. 9, 2013; doi:10.1038/nature11814

Contact: Darrin D. Stuart, Novartis Institutes for BioMedical Research, Emeryville, Calif.

e-mail: darrin.stuart@novartis.com

Contact: Martin McMahon, University of California, San Francisco, Calif.

e-mail: mcmahon@cc.ucsf.edu

2. Flaherty, K.T. et al. N. Engl. J. Med. 363, 809-819 (2010)

3. Nazarian, R. et al. Nature 468, 973-977 (2010)
4. Wagle, N. et al. J. Clin. Oncol. 29, 3085-3096 (2011)

5. Jänne, P.A. et al. Nat. Rev. Drug Discov. 8, 709-723 (2009)

COMPANIES AND INSTITUTIONS MENTIONED

American Society of Clinical Oncology, Alexandria, Va.

Daiichi Sankyo Co. Ltd. (Tokyo:4568; Osaka:4568), Tokyo, Japan

GlaxoSmithKline plc (LSE:GSK; NYSE:GSK), London, U.K.

Novartis Institutes for BioMedical Research, Emeryville, Calif.

Roche (SIX:ROG; OTCQX:RHHBY), Basel, Switzerland

University of California, San Francisco, Calif. 